\title{
First-principles description of charge transfer in donor-acceptor compounds from self-consistent many-body perturbation theory
}

\author{
Fabio Caruso, ${ }^{1,2}$ Viktor Atalla, ${ }^{1}$ Xinguo Ren, ${ }^{3}$ Angel Rubio,,${ }^{1,4,5}$ Matthias Scheffler, ${ }^{1}$ and Patrick Rinke ${ }^{1}$ \\ ${ }^{1}$ Fritz-Haber-Institut der Max-Planck-Gesellschaft, Faradayweg 4-6, D-14195, Berlin, Germany \\ ${ }^{2}$ Department of Materials, University of Oxford, Parks Road, Oxford OX1 3PH, United Kingdom \\ ${ }^{3}$ Key Laboratory of Quantum Information, University of Science and Technology of China, Hefei 230026, China \\ ${ }^{4}$ Nano-Bio Spectroscopy Group and ETSF Scientific Development Centre, Departamento Física de Materiales, \\ Universidad del País Vasco, CFM CSIC-UPV/EHU-MPC and DIPC, Av. Tolosa 72, E-20018, San Sebastián, Spain \\ ${ }^{5}$ European Theoretical Spectroscopy Facility \\ (Received 27 June 2014; published 26 August 2014)
}

\begin{abstract}
We investigate charge transfer in prototypical molecular donor-acceptor compounds using hybrid density functional theory (DFT) and the $G W$ approximation at the perturbative level $\left(G_{0} W_{0}\right)$ and at full self-consistency (sc-GW). For the systems considered here, no charge transfer should be expected at large intermolecular separation according to photoemission experiments and accurate quantum-chemistry calculations. The capability of hybrid exchange-correlation functionals of reproducing this feature depends critically on the fraction of exact exchange $\alpha$, as for small values of $\alpha$ spurious fractional charge transfer is observed between the donor and the acceptor. $G_{0} W_{0}$ based on hybrid DFT yields the correct alignment of the frontier orbitals for all values of $\alpha$. However, $G_{0} W_{0}$ has no capacity to alter the ground-state properties of the system because of its perturbative nature. The electron density in donor-acceptor compounds thus remains incorrect for small $\alpha$ values. In sc- $G W$, where the Green's function is obtained from the iterative solution of the Dyson equation, the electron density is updated and reflects the correct description of the level alignment at the $G W$ level, demonstrating the importance of self-consistent many-body approaches for the description of ground- and excited-state properties in donor-acceptor systems.
\end{abstract}

DOI: 10.1103/PhysRevB.90.085141

PACS number(s): 31.15.-p, 71.10.-w, 71.15.Qe

\section{INTRODUCTION}

Donor-acceptor compounds have recently attracted considerable attention due to their application in the field of organic electronics [1,2]. A description of donor-acceptor complexes from first principles is desirable to achieve an atomistic understanding of charge-transfer processes and their impact on electronic properties. However, charge transfer remains a major challenge for presently available first-principles techniques [3,4].

In the weak-coupling limit (i.e., when the wave-function overlap between the donor and the acceptor becomes negligible), the lowest charge-transfer energy $\left(E_{\mathrm{CT}}\right)$ is determined by the highest-occupied molecular orbital (HOMO) of the donor and the lowest-unoccupied molecular orbital (LUMO) of the acceptor. The HOMO and LUMO energies are equal to the negative of the ionization potential (IP) and the electron affinity (EA). In exact density functional theory (DFT) these values are given by the highest-occupied Kohn-Sham (KS) levels of the $N$ and $N+1$ electron systems. For approximate DFT, the SlaterJanak transition states [5], i.e., the KS levels of the $N-\frac{1}{2}$ and $N+\frac{1}{2}$ electron systems, provide an accurate estimate of the HOMO and LUMO energies. At large separation between donor and acceptor, charge transfer may occur in the ground state if the HOMO of the donor lies energetically above the LUMO of the acceptor or as a neutral charge transfer excitation otherwise. Therefore, first-principles methods that do not accurately capture orbital energies of the $N-\frac{1}{2}$ and $N+\frac{1}{2}$ electron systems may provide a qualitatively incorrect description of charge transfer and, subsequently, ground-state properties such as the charge density.
An alternative to DFT for the description of the HOMO and LUMO (or IP and EA) energies is many-body perturbation theory. Below we apply many-body Green's function theory to describe charge transfer. The single-particle Green's function provides a rigorous way to determine electronic excitations in molecules and solids and gives access to the total energy and therefore the ground-state properties of a system. In this context, Hedin's $G W$ approximation [6] for the single-particle Green's function has become a well-established framework for the calculation of IP and EA, also referred to as quasiparticle excitations [7-9]. However, in perturbative $G W$ calculations $\left(G_{0} W_{0}\right)[10]$ only the quasiparticle energies are evaluated at the $G W$ level, whereas the ground-state density is left unchanged and remains at the unperturbed level, typically DFT. If spurious charge transfer has occurred at the DFT stage due to an inherent deficiency of the chosen exchange-correlation (XC) functional, $G_{0} W_{0}$ cannot rectify this charge transfer, despite the fact that $G_{0} W_{0}$ may yield a qualitatively correct HOMOLUMO alignment in donor-acceptor systems.

In this work, we demonstrate that the self-consistent $G W$ approach (sc- $G W$ ) — in which the Green's function is obtained from the iterative solution of the Dyson equation-provides a suitable first-principles framework for the description of donor-acceptor systems. Compared to $G_{0} W_{0}$, the main advantages of the sc- $G W$ method are the consistent description of ground and excited states and its independence of the initial reference ground state [11]. For a set of prototypical donor-acceptor complexes, we assess the performance of DFT hybrid exchange-correlation functionals, $G_{0} W_{0}$, and sc- $G W$ based on the following criteria: (i) accuracy of the quasiparticle spectrum and (ii) charge transfer. We show that sc- $G W$ yields 
a qualitatively correct HOMO-LUMO alignment. Moreover, it correctly predicts that the chosen donor-acceptor complexes do not exhibit any charge transfer at large donor-acceptor distances, as expected from reference experimental data and high-level quantum-chemical calculations.

The remainder of this article is organized as follows. In Sec. II, we give an overview of the theoretical and computational methods employed in this work. Section III introduces the problem of charge transfer in donor-acceptor complexes in DFT and $G W$-based approaches. The origin of charge transfer in DFT and in $G W$ is discussed in Secs. IV and V, respectively. Finally, a summary and conclusions are reported in Sec. VI.

\section{COMPUTATIONAL APPROACH}

In this work, we apply the Perdew-Burke-Ernzerhof (PBE) hybrid family of $\mathrm{XC}$ functionals $[12,13]$, which expresses the $\mathrm{XC}$ energy as

$$
E_{\mathrm{xc}}=\alpha E_{\mathrm{x}}^{\mathrm{EX}}+(1-\alpha) E_{\mathrm{x}}^{\mathrm{PBE}}+E_{\mathrm{c}}^{\mathrm{PBE}},
$$

where $E_{\mathrm{x}(\mathrm{c})}^{\mathrm{PBE}}$ is the PBE exchange (correlation) energy, $E_{\mathrm{x}}^{\mathrm{EX}}$ the exact exchange (EX) energy, and $\alpha \in[0,1]$ a real parameter. As an example, the PBE0 functional is obtained by setting $\alpha=1 / 4$ in Eq. (1).

In $G_{0} W_{0}$, the quasiparticle energies $\epsilon_{n}^{\mathrm{QP}}$ are obtained from the first-order perturbative correction of the generalized KohnSham (GKS) eigenvalues $\epsilon_{n}^{0}$ :

$$
\epsilon_{n}^{\mathrm{QP}}=\epsilon_{n}^{0}+\left\langle\psi_{n}\left|\hat{\Sigma}\left(\epsilon_{n}^{\mathrm{QP}}\right)-\hat{v}_{\mathrm{xc}}\right| \psi_{n}\right\rangle,
$$

where $\hat{\Sigma}$ is the $G_{0} W_{0}$ self-energy, $\hat{v}_{\text {xc }}$ the XC potential of the preceding calculation, and $\psi_{n}$ the GKS orbitals. Here and below, spin indices have been omitted for simplicity. In $G_{0} W_{0}$, the quasiparticle correction is applied only to the eigenvalues, whereas ground-state properties remain unaffected. The perturbative inclusion of higher-order terms in the self-energy [such as second-order screened exchange (SOSEX), or vertex corrections [14-16]] are expected to improve the agreement of the computed excitation energies with the experiment, but similar to $G_{0} W_{0}$, they would have no effect on the ground-state properties of the systems. To incorporate the effect of the self-energy into the ground state, self-consistency is essential. In sc- $G W$ the Green's function $G$ is updated by solving the Dyson equation iteratively

$$
G(\epsilon)=G_{0}(\epsilon)+G_{0}(\epsilon)\left[\Sigma(\epsilon)+\Delta v_{\mathrm{H}}-v_{\mathrm{xc}}\right] G(\epsilon),
$$

where $G_{0}$ is the Green's function of the DFT reference system and $\Delta v_{\mathrm{H}}$ the difference of the $G W$ and DFT Hartree potentials. At self-consistency, quasiparticle excitation energies are extracted directly from the spectral function $A(\omega)=1 / \pi|\operatorname{Tr}[\operatorname{Im} G(\omega)]|$. Additionally, from the selfconsistent Green's function one may derive ground-state properties that are consistent with the $G W$ self-energy as, for instance, the electron density

$$
n(\mathbf{r})=-i G\left(\mathbf{r}, \mathbf{r}, \tau=0^{-}\right),
$$

where $\tau$ denotes imaginary time (see Ref. [17] for details). charge-transfer excitation

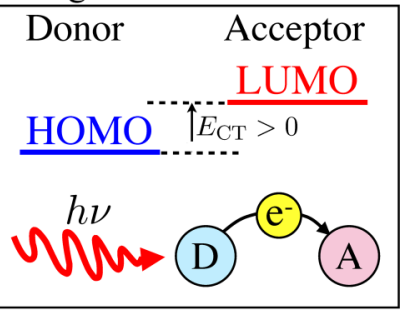

level alignment before electron transfer

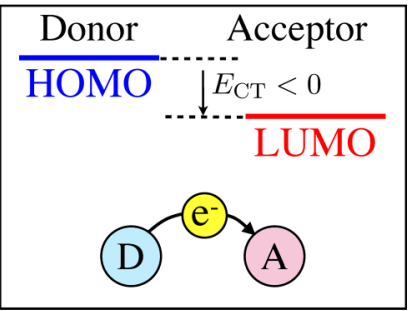

FIG. 1. (Color online) Schematic representation of the level alignment in weakly interacting donor-acceptor compounds. For $E_{\mathrm{CT}}>0$ (left), charge transfer occurs as an excitation (e.g., upon absorption of a photon with energy $h v \geqslant E_{\mathrm{CT}}$ ). For negative values of the charge-transfer energy (right), the system is characterized by charge transfer in the ground state.

\section{DONOR-ACCEPTOR COMPLEXES}

In the following, we consider prototypical donor-acceptor systems obtained from a cofacial arrangement of donor and acceptor molecules. In particular, we choose tetrathiafulvalene (TTF) as the donor molecule and three different acceptors: tetracyanoethylene (TCNE), tetracyanoquinodimethane (TCNQ), and p-chloranil. All calculations are performed with the all-electron numeric atom-centered orbital code FHIAIMS [17-19]. The geometries of the individual molecules are obtained from a PBE geometry optimization performed with FHI-AIMS' Tier 2 basis set. For more details on the FHI-AIMS basis sets please see Refs. [18-20]. All geometries are listed in Appendix B. For brevity, the following discussion is centered on the TTF-TCNE dimer.

\section{A. TTF-TCNE dimer}

For small weakly interacting molecules, the charge-transfer energy at large intermolecular distances $R$ may be approximated by

$$
E_{\mathrm{CT}}=\mathrm{IP}(\text { donor })-\mathrm{EA}(\text { acceptor })-\frac{f^{2}}{R} .
$$

The last term is the Coulomb interaction arising from the transfer of $f$ electrons from the donor to the acceptor. These two situations are schematically illustrated in Fig. 1.

In the weak-coupling limit $(R \rightarrow \infty)$ the Coulomb term can be neglected and Eq. (5) reduces to

$$
E_{\mathrm{CT}}=\mathrm{IP}(\text { donor })-\mathrm{EA}(\text { acceptor }) \text {, }
$$

which now only depends on the relative energy position between the IP of the neutral donor and the EA of the neutral acceptor. Charge transfer between the monomers occurs in the ground state whenever $E_{\mathrm{CT}}$ is negative [EA(acceptor) $>$ $\mathrm{IP}$ (donor)], whereas positive values of $E_{\mathrm{CT}}$ [EA(acceptor) < $\mathrm{IP}($ donor$)]$ indicate charge-transfer excitations. In this limitaccording to experiment and coupled-cluster singles doubles with perturbative triples calculations $[\operatorname{CCSD}(\mathrm{T})]$ for the IP of TTF and the EAs of the donors considered here (see Table I)_no charge transfer should be expected at large intermolecular separation since $E_{\mathrm{CT}}>0$ for all donor-acceptor pairs. 
TABLE I. Comparison of experimental IPs and EAs with HOMO and LUMO energies of TTF and TCNE from different levels of theory.

\begin{tabular}{lccc}
\hline \hline & HOMO $_{\text {TTF }}$ & LUMO $_{\text {TCNE }}$ & $E_{\mathrm{CT}}$ \\
\hline PBE & -3.94 & -5.84 & -1.9 \\
PBE0 & -4.81 & -5.20 & -0.39 \\
PBEh $\left(\alpha^{*}\right)$ Ref. [22] & -6.82 & -3.51 & 3.31 \\
PBEh $(\bar{\alpha})$ Ref. [23] & -4.28 & -5.33 & -1.05 \\
$G_{0} W_{0} @$ PBE & -6.21 & -3.85 & 2.36 \\
$G_{0} W_{0} @ P B E 0$ & -6.42 & -3.70 & 2.72 \\
$G_{0} W_{0} @$ PBEh $\left(\alpha^{*}\right)$ Ref. [22] & -6.82 & -3.54 & 3.28 \\
$G_{0} W_{0} @$ PBEh $(\bar{\alpha})$ Ref. [23] & -6.28 & -3.77 & 2.51 \\
sc- $G W$ & -5.96 & -3.90 & 2.06 \\
Exp. Ref. [24] & -6.70 & & $(3.76)$ \\
CCSD $(\mathrm{T}) \operatorname{Ref} .[25]$ & & -2.94 & $(3.76)$ \\
\hline \hline
\end{tabular}

We first address the ground-state properties of the TTFTCNE dimer. For a quantitative assessment of the charge transfer between the donor and acceptor, we evaluated the dipole moment of the TTF-TCNE dimer for several values of the intermolecular distance (right panel of Fig. 2). Since charge transfer should not take place in the weak-coupling limit, the component of the dipole moment parallel to the TTF-TCNE axis is expected to vanish. For PBE calculations and PBE-based hybrid functionals with $\alpha<0.3$, however, we observe a linear divergence of the dipole moment for increasing distance between the monomers. The diverging dipole at large intermolecular separation is a clear indication that charge is transferred from the donor to the acceptor. The charge transfered between TTF and TCNE-estimated from the ratio between the dipole moment and the intermolecular distance (left panel of Fig. 2) - demonstrates that in PBE approximately one-fourth of an electron is transferred from TTF to TCNE. This picture is unaffected by the $G_{0} W_{0}$ quasiparticle correction of Eq. (2) because $G_{0} W_{0}$ only corrects the DFT levels a posteriori, but not the electron density which eventually determines the dipole moment. On the other hand, the sc- $G W$ dipole moment-derived from the sc- $G W$ density—vanishes at large separation between the monomers

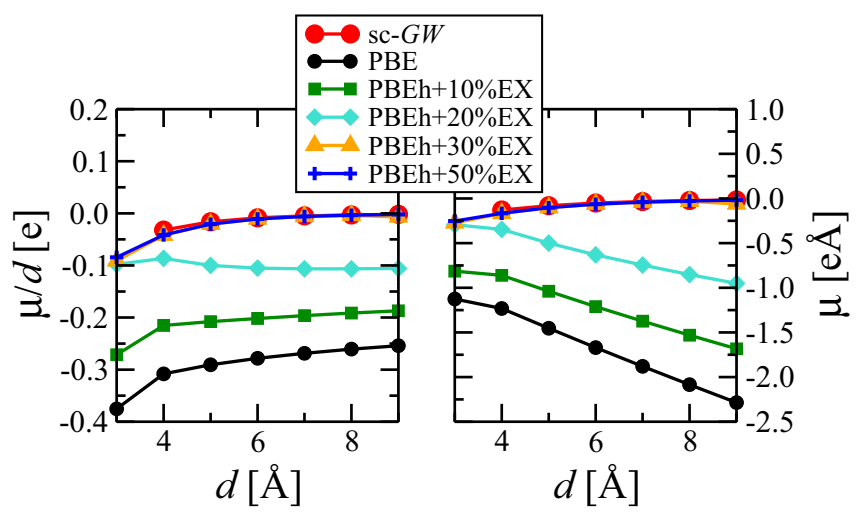

FIG. 2. (Color online) Left: The charge difference between TTF and TCNE is estimated from the ratio between the dipole moment and the distance between the centers of two molecules. Right: Dipole moment of the TTF-TCNE dimer as a function of the intermolecular distance.

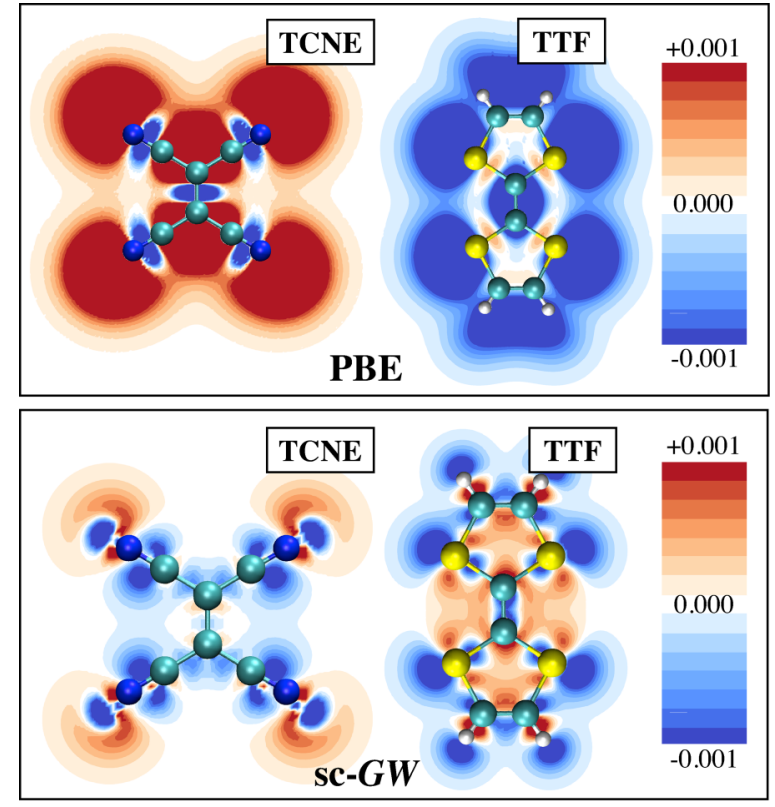

FIG. 3. (Color online) Volume slices of the density difference between the TTF-TCNE dimer at $5 \AA$ distance and the monomers in PBE (upper panel) and sc- $G W$ (lower panel). Units are $\AA^{-3}$.

and thus demonstrates that charge transfer between the donor and the acceptor is zero in the weak-coupling limit. On the DFT side, a charge-transfer-free description can be obtained from hybrid functionals that use a high $\alpha$ value (>0.3). For a more detailed discussion of charge transfer in DFT we refer to Sec. IV and Ref. [21].

To illustrate the changes in the ground-state density induced by spurious fractional charge transfer, we report in Fig. 3 volume slices of the difference between the TTF-TCNE density and the density of the isolated (neutral) monomers, evaluated from PBE (above) and sc- $G W$ (below) at a distance of $5 \AA$. The PBE density difference is mostly positive (red) on TCNE and negative (blue) on TTF. It therefore manifests an accumulation of electron density on the acceptor accompanied by a charge depletion on the donor. In sc- $G W$, however, the electron density is only slightly perturbed due to the weak interaction between the monomers. The density difference does not exhibit any charge transfer between TTF and TCNE.

\section{B. TTF-TCNQ dimer}

An additional example is provided by the TTF-TCNQ dimer shown in Fig. 4. The geometry of the dimer is taken from an interface between TTF and TCNQ crystals along the [001] surface of TCNQ, in analogy to the work presented in Ref. [22]. Figure 4 reports isosurfaces of the density difference between the dimer and the monomers. Similar to the TTF-TCNE dimer, the highest-occupied KS level of the isolated TTF molecule $(-3.94 \mathrm{eV})$ incorrectly lies above the lowest-unoccupied KS level of TCNQ $(-5.59 \mathrm{eV})$ in PBE. As a result, a fraction of an electron is transferred from TTF to TCNQ. In sc- $G W$ no charge transfer occurs, in analogy with the TTF-TCNE dimer discussed before. However, we observe a small charge rearrangement where the molecules are closest. This is most likely due to the Pauli principle, which requires that the 


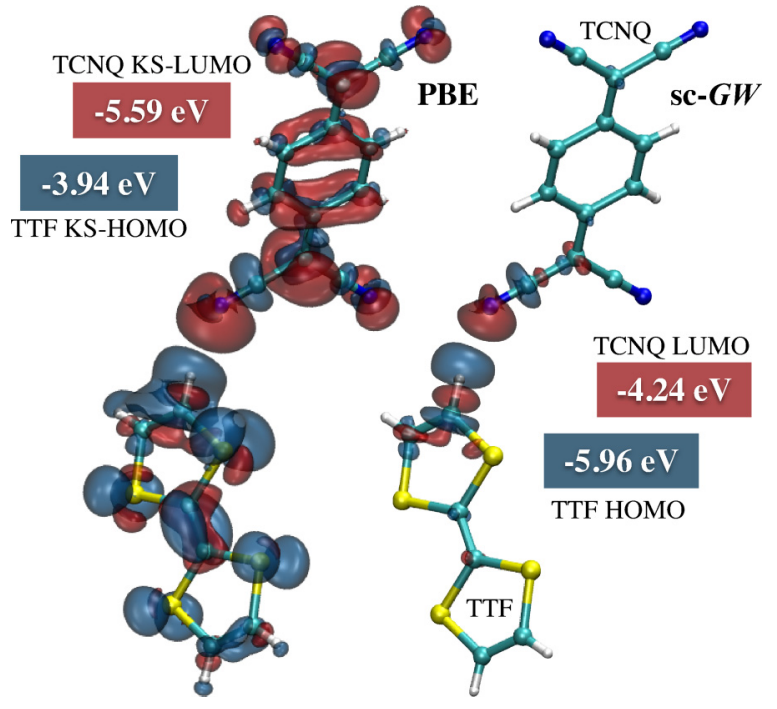

FIG. 4. (Color online) Density difference between the TTFTCNQ dimer and the monomers for PBE (left) and sc- $G W$ (right). Red (blue) isosurfaces indicate charge accumulation (depletion) resulting from the TTF-TCNQ level alignment.

molecular states of each molecule also have to be orthogonal to the states of the other molecule.

\section{CHARGE TRANSFER IN DFT}

The spurious charge transfer that PBE and hybrid functionals with a low fraction of exact exchange predict for donor-acceptor systems is related to the deviation from straight line error (DSLE). In other words, the total energy does not exhibit a linear behavior for fractional electron numbers, as expected for the exact XC functional. Janak's theorem [5] establishes a relation between the Kohn-Sham eigenvalues and the total energy of a system with fractional electron number. For instance, for the HOMO level $\left(\epsilon^{\mathrm{H}}\right)$ one has $\partial E(f) / \partial f=\epsilon^{\mathrm{H}}(f)$, where $E(f)$ denotes the total energy of a system in which the highest-occupied KS level is occupied with $f$ electrons (with $N_{0}-1<f \leqslant N_{0}, N_{0}$ being the integer number of electrons in the neutral system). The corresponding eigenvalue of the molecule at integer occupation is then obtained in the limit $f \rightarrow N_{0}^{-}$. If the total energy is a linear function of $f$, the $\mathrm{KS}$ eigenvalue (i.e., the derivative of the total energy) is independent of $f$. Conversely, if the $\mathrm{XC}$ functional suffers from DSLE, the highest-occupied and lowest-unoccupied KS levels will exhibit an $f$ dependence, which will be stronger for larger deviations from linearity. For XC functionals that produce a convex deviation from the straight line, the highest-occupied KS level is too low in energy and the lowest-unoccupied KS level too high. For a concave deviation, such as in PBE, the highest-occupied $\mathrm{KS}$ level is too high and the lowest-unoccupied KS level too low. A concave deviation could thus result in spurious charge transfer [21].

Hybrid functionals can be used to reduce or eliminate the DSLE. We achieve this by varying the fraction $\alpha$ of exact exchange in the PBEh hybrid functional until the DSLE is minimized [21]. The resulting values $\left(\alpha^{\dagger}\right)$ can be found in Table II.
TABLE II. Optimized $\alpha$ values of TTF, TCNE, TCNQ, and pchloranil determined according to Refs. [22] $\left(\alpha^{*}\right)$ and [23] $(\bar{\alpha})$.

\begin{tabular}{lcccc}
\hline \hline & TTF & TCNE & TCNQ & p-chloranil \\
\hline $\bar{\alpha}$ (Ref. [23]) & 0.10 & 0.20 & 0.24 & 0.08 \\
$\alpha^{*}$ (Ref. [22]) & 0.78 & 0.83 & 0.81 & 0.74 \\
$\alpha^{\dagger}$ (Ref. [21]) & 0.7 & - & 0.7 & - \\
\hline \hline
\end{tabular}

\section{CHARGE TRANSFER IN $G W$}

Figure 5 reports $E_{\mathrm{CT}}$ for $G_{0} W_{0}$ calculations based on $\mathrm{PBEh}$ hybrid calculations as a function of $\alpha$. For comparison, $E_{\mathrm{CT}}$ for PBEh itself is also shown. For low $\alpha E_{\mathrm{CT}}$ is negative, which is consistent with the spurious charge transfer found in DSLE functionals. $E_{\mathrm{CT}}$ increases linearly with $\alpha$ and approaches the experimental value for $\alpha$ values close to $\alpha^{\dagger}$.

In contrast, $G_{0} W_{0}$ calculations based on PBEh always yield positive charge transfer energies $E_{\mathrm{CT}}$ that are in quantitative agreement with the reference data for all values of $\alpha$ (see, e.g., Table I and Fig. 5). However, since $G_{0} W_{0}$ calculations only correct the DFT eigenvalues, the $G_{0} W_{0}$ approach cannot repair the occurrence of spurious charge transfer. To achieve a qualitatively correct description of both ground- and excitedstate properties of donor-acceptor systems in $G_{0} W_{0}$, it is therefore essential to base $G_{0} W_{0}$ on hybrid functionals with large $\alpha$ values.

These results illustrate that the choice of $\alpha$ in Eq. (1) is critical for the accuracy of ground-state properties. Similarly, it has been demonstrated that the accuracy achievable in the description of $G_{0} W_{0}$ quasiparticle excitation energies also exhibits a strong $\alpha$ dependence [22,23,26]. To ameliorate this shortcoming, several strategies for a first-principles determination of $\alpha$ based on the $G_{0} W_{0}$ approximation have been suggested recently. In Ref. [22], the authors proposed to determine $\alpha$ by minimizing the $G_{0} W_{0}$ quasiparticle correction of the highest-occupied KS level (later referred to as the $\alpha^{*}$ method). In their consistent starting point approach (CSP), Körzdörfer and Marom [23] suggested to determine $\alpha$ such

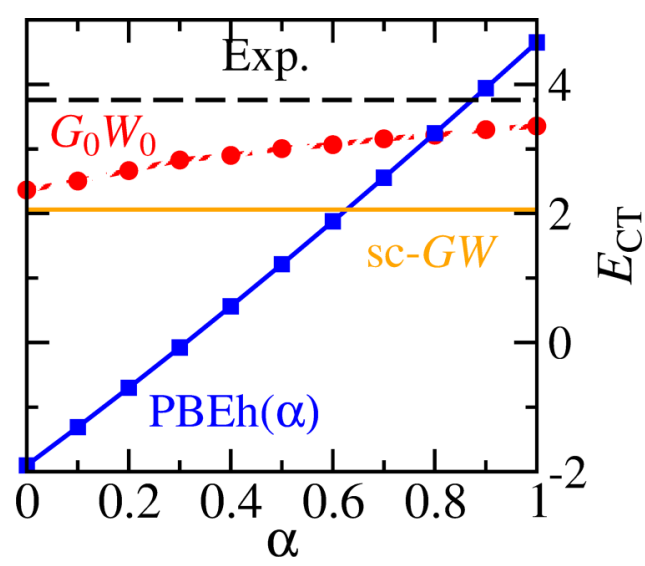

FIG. 5. (Color online) $G_{0} W_{0} @ \operatorname{PBEh}(\alpha)$ and sc- $G W$ values for $E_{\mathrm{CT}}$ as a function of $\alpha$. The $\operatorname{PBEh}(\alpha)$ values are estimated from the difference between the lowest-unoccupied and highest-occupied KS levels. The difference between the experimental IP of TTF and EA of TCNE is included for comparison. 
that the hybrid functional eigenvalue spectrum is as close as possible to a rigid shift of the $G_{0} W_{0}$ spectrum for the valence states. Their $\alpha$ value is denoted $\bar{\alpha}$ in the following. Alternatively, $\alpha$ may be chosen to enforce the Koopmans' condition [27] by requiring that the highest-occupied KS level energy agrees with the total-energy difference between the neutral and the singly ionized system (i.e., the $\Delta$-selfconsistent field, or $\Delta$-SCF, ionization energy). However, due to the qualitative agreement of the $G_{0} W_{0}$ and $\Delta$-SCF ionization energies, the latter method is expected to yield $\alpha$ values similar to the $\alpha^{*}$ method of Ref. [22].

For the donor and acceptors considered here, the $\alpha^{*}$ and $\bar{\alpha}$ values are reported in Table II. For TTF and TCNE, the $\alpha^{*}$ method yields $\alpha^{*}=0.78$ and $\alpha^{*}=0.83$, respectively. The CSP approach gives $\bar{\alpha}=0.10$ for TTF and $\bar{\alpha}=0.20$ for TCNE. For both approaches, the tuned hybrid parameter for the TTF-TCNE dimer was obtained from an average of the coefficients of the isolated molecules, i.e., $\alpha^{*}=0.8$ and $\bar{\alpha}=0.15$. Due to the large fraction of EX, the $\alpha^{*}$ method produces the correct level alignment in donor-acceptor complexes. Moreover, the highest-occupied (lowest-unoccupied) KS levels of TTF (TCNE) and $E_{\mathrm{CT}}$ agree with the experimental reference values (Table I). The CSP approach, on the other hand, generally yields smaller $\alpha$ values (for the systems considered here $0.1<\bar{\alpha}<0.3$ ), which do not recover the correct level alignment between the donor and the acceptor. Therefore, the associated hybrid functional ground state is still characterized by spurious charge transfer.

sc- $G W$, like $G_{0} W_{0}$, always predicts positive charge-transfer energies although the sc- $G W$ HOMO (LUMO) energy of TTF (TCNE) is slightly less accurate than the corresponding quantity in $G_{0} W_{0}$ (see, e.g., Table I). However, the Green's function obtained from the solution of the Dyson equation, Eq. (3), is independent of the starting point and, therefore, of the EX parameter $\alpha$ (Fig. 5). In addition, the electron density is also updated through Eq. (4). Therefore, in contrast to $G_{0} W_{0}$, the ground-state properties are consistent with the correct level alignment, i.e., they are charge-transfer free. We notice, however, that sc- $G W$ has a tendency to underestimate (overestimate) the IP (EA) for TTF-TCNE. The resulting $E_{\mathrm{CT}}$ is underestimated by approximately $2 \mathrm{eV}$ compared to the reference value, showing that sc- $G W$ may still yield spurious ground-state charge transfer for donor-acceptor systems with small positive charge-transfer energies.

Monitoring the frontier-orbital energies of the TTF-TCNE dimer as a function of distance between the monomers (Fig. 6, left) reveals an additional failure of the perturbative $G_{0} W_{0}$ approach. For all intermolecular separations, PBE yields degenerate highest-occupied and lowest-unoccupied KS levels. For distances larger than $3 \AA, G_{0} W_{0}$ calculations break the HOMO-LUMO degeneracy of the PBE starting point and yield positive charge-transfer energies (Fig. 6, right). However, for small distances, $G_{0} W_{0} @ \mathrm{PBE}$ also yields vanishing charge-transfer energies and, therefore, might provide an overestimation of the charge transfer between donors and acceptors. In sc- $G W$, on the other hand, the separation between the HOMO and LUMO always remains finite and, correspondingly, $E_{\mathrm{CT}}>0$ at all distances.

We next discuss the accuracy of the quasiparticle excitation spectrum of the donor and acceptor molecules. For
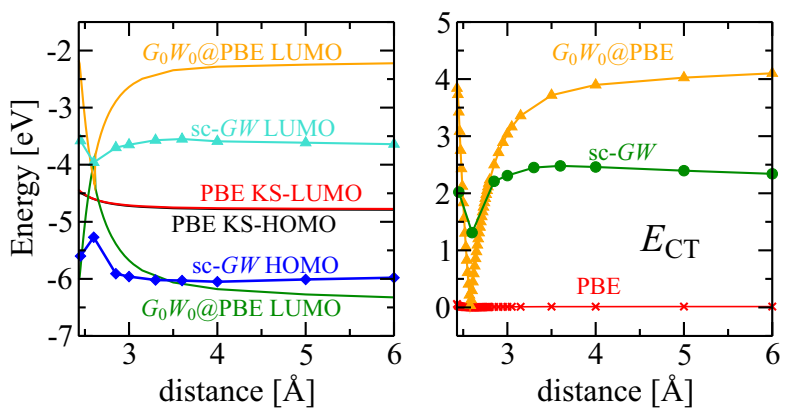

FIG. 6. (Color online) Left: PBE, $G_{0} W_{0} @ \mathrm{PBE}$, and sc- $G W$ HOMO and LUMO levels for the TTF-TCNE dimer as a function of the distance between the monomers. Right: $E_{\mathrm{CT}}$ as a function of distance.

TTF, TCNE, TCNQ, and p-chloranil, the mean absolute error (MAE) and mean error (ME) of the $G_{0} W_{0} @ \operatorname{PBEh}(\alpha)$ quasiparticle energies relative to photoemission experiment for the HOMO level and for the valence excitation spectrum are reported in Fig. 7 for $\alpha \in[0,1]$. The individual numbers are given in Table III in the Appendix. For the valence states, the MAE and ME refer to the first $N$ excitation energies ( $N=10$ for TCNE and TCNQ, $N=6$ for TTF, $N=7$ for p-chloranil) for which experimental data are available. The best agreement with experiment-with a MAE of about $0.2 \mathrm{eV}$ - is obtained with $\alpha=0.35$ for the HOMO, and $\alpha=$ 0.4 if all experimentally available excitation energies are considered. The sc- $G W$ ionization energies have a MAE of $0.4 \mathrm{eV}$, which - being independent from the choice of $\alpha$--provides an unbiased assessment of the accuracy of the $G W$ approximation for the excitation spectrum of these systems. For $\alpha>0.5$ the MAE is considerably larger for the valence spectrum than for the HOMO alone, indicating that large fractions of EX deteriorate the description of lower-lying excitations significantly, as demonstrated previously for benzene and the azabenzenes [26]. The increase of the MAE for large $\alpha$ (and similarly for small $\alpha$ ) values may be justified in terms of the underscreening of the screened Coulomb interaction $W$

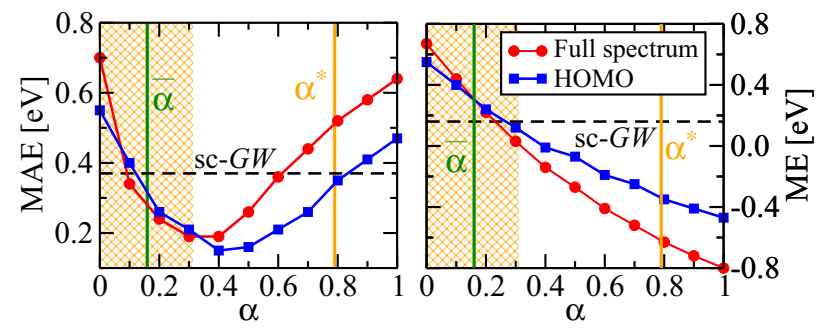

FIG. 7. (Color online) Mean absolute error (MAE, left) and mean error (ME, right) relative to experiments [28-31] of the $G_{0} W_{0} @ \operatorname{PBEh}(\alpha)$ quasiparticle energies of TTF, TCNE, TCNQ, and p-chloranil as a function of $\alpha$ for the HOMO level (squares) and the full excitation spectrum (circles). The dashed lines refer to the sc- $G W$ errors for the full excitation spectrum. The (average) optimally tuned parameters $\alpha^{*}$ and $\bar{\alpha}$-determined according to Refs. [22] and [23], respectively - are reported as vertical solid lines, whereas the shaded region indicates the range of $\alpha$ yielding spurious asymptotic charge transfer for TTF and TCNE. 
TABLE III. Mean absolute error (MAE) of the sc- $G W$ and $G_{0} W_{0}$ quasiparticle energies based on different starting points as compared to the first $N$ IPs ( $N=10$ for TCNE and TCNQ, $N=6$ for TTF, $N=7$ for p-chloranil) experimentally available from Refs. [28-31].

\begin{tabular}{lccccc}
\hline \hline & TCNE & TCNQ & P-chloranil & TTF & Average \\
\hline sc- $G W$ & 0.30 & 0.36 & 0.42 & 0.42 & 0.37 \\
$G_{0} W_{0} @ \mathrm{PBE}$ & 1.03 & 0.91 & 0.35 & 0.51 & 0.70 \\
$G_{0} W_{0} @ \mathrm{PBE} 0$ & 0.35 & 0.38 & 0.38 & 0.16 & 0.32 \\
$G_{0} W_{0} @ \mathrm{HF}$ & 0.84 & 0.66 & 1.41 & 0.61 & 0.88 \\
$G_{0} W_{0} @ \operatorname{PBEh}\left[\alpha^{*}\right][22]$ & 0.58 & 0.42 & 1.06 & 0.39 & 0.61 \\
$G_{0} W_{0} @ \operatorname{PBEh}[\bar{\alpha}][23]$ & 0.48 & 0.40 & 0.25 & 0.37 & 0.37 \\
$G_{0} W_{0} @ \operatorname{PBEh}(\alpha=0.2)$ & 0.48 & 0.36 & 0.30 & 0.20 & 0.24 \\
$G_{0} W_{0} @ \operatorname{PBEh}(\alpha=0.4)$ & 0.05 & 0.23 & 0.61 & 0.09 & 0.19 \\
$G_{0} W_{0} @ \operatorname{PBEh}(\alpha=0.6)$ & 0.30 & 0.41 & 0.90 & 0.24 & 0.36 \\
$G_{0} W_{0} @ \operatorname{PBEh}(\alpha=0.8)$ & 0.56 & 0.62 & 1.16 & 0.36 & 0.52 \\
$G_{0} W_{0} @ \operatorname{PBEh}(\alpha=1.0)$ & 0.74 & 0.79 & 1.37 & 0.46 & 0.64 \\
\hline \hline
\end{tabular}

arising from the overstretching of the Kohn-Sham spectrum. The effect of overscreening and underscreening is clearly illustrated by the quasilinear $\alpha$ dependence of the ME of $G_{0} W_{0}$ calculations (Fig. 7, right), indicating that quasiparticle energies are underestimated (overestimated) for small (large) $\alpha$ values.
TABLE IV. PBE-optimized geometry of p-chloranine in Cartesian coordinates and $\AA$.

\begin{tabular}{lrrr}
\hline \hline p-chloranine & & & \\
\hline $\mathrm{C}$ & -1.86581 & 2.17291 & -0.04169 \\
$\mathrm{C}$ & -0.93853 & 3.13223 & 0.04182 \\
$\mathrm{C}$ & 0.50931 & 2.80448 & 0.11635 \\
$\mathrm{C}$ & 0.88471 & 1.36593 & 0.09443 \\
$\mathrm{C}$ & -0.04258 & 0.40661 & 0.01091 \\
$\mathrm{C}$ & -1.49041 & 0.73437 & -0.06380 \\
$\mathrm{O}$ & 1.36033 & 3.68495 & 0.19285 \\
$\mathrm{O}$ & -2.34142 & -0.14609 & -0.14059 \\
$\mathrm{Cl}$ & -3.56355 & 2.51305 & -0.13043 \\
$\mathrm{Cl}$ & -1.33437 & 4.82019 & 0.07037 \\
$\mathrm{Cl}$ & 0.35325 & -1.28135 & -0.01743 \\
$\mathrm{Cl}$ & 2.58243 & 1.02579 & 0.18338 \\
\hline \hline
\end{tabular}

\section{ACKNOWLEDGMENTS}

This work was supported by the European Research Council Advanced Grant DYNamo (ERC-2010-AdG-267374), the European Commission within the FP7 CRONOS project (ID 280879), Spanish Grant (FIS2010-21282-C02-01), Ikerbasque and Grupos Consolidados UPV/EHU del Gobierno Vasco (IT578-13).

\section{APPENDIX A: MEAN ABSOLUTE ERRORS OF THE IONIZATION ENERGIES}

In Table III we report the mean absolute errors (MAE) for the ionization energies of TTF, TCNE, p-chloranil, and TCNQ. The MAE refers to the first $N$ excitation energies $(N=10$ for TCNE and TCNQ, $N=6$ for TTF, $N=7$ for p-chloranil) for which experimental data are available [29,31-33].

\section{APPENDIX B: MOLECULAR GEOMETRIES}

Tables IV to VII summarize the geometries of p-chloranine, TTF, TCNQ, and TCNE optimized in the PBE approximation.

TABLE V. PBE-optimized geometry of TTF in Cartesian coordinates and $\AA$.

\begin{tabular}{lrrr}
\hline \hline TTF & & & \\
\hline S & -1.64052 & -1.48559 & 0.00163 \\
S & -1.64039 & 1.48575 & 0.00150 \\
S & 1.64051 & 1.48558 & 0.00164 \\
S & 1.64036 & -1.48575 & 0.00152 \\
$\mathrm{C}$ & -0.67944 & 0.00003 & 0.00113 \\
$\mathrm{C}$ & 0.67942 & -0.00003 & 0.00113 \\
$\mathrm{C}$ & -3.18226 & -0.67153 & -0.00158 \\
$\mathrm{C}$ & -3.18220 & 0.67182 & -0.00164 \\
$\mathrm{C}$ & 3.18225 & 0.67151 & -0.00157 \\
$\mathrm{C}$ & 3.18218 & -0.67184 & -0.00164 \\
$\mathrm{H}$ & -4.07491 & -1.29252 & -0.00288 \\
$\mathrm{H}$ & -4.07479 & 1.29289 & -0.00302 \\
$\mathrm{H}$ & 4.07490 & 1.29250 & -0.00288 \\
$\mathrm{H}$ & 4.07477 & -1.29291 & -0.00302 \\
\hline \hline
\end{tabular}


TABLE VI. PBE-optimized geometry of TCNQ in Cartesian coordinates and $\AA$.

\begin{tabular}{lrrr}
\hline \hline TCNQ & & & \\
\hline $\mathrm{C}$ & 0.00000 & 1.42000 & 0.00000 \\
$\mathrm{C}$ & -1.23502 & 0.68052 & 0.00000 \\
$\mathrm{C}$ & 1.23502 & 0.68052 & 0.00000 \\
$\mathrm{C}$ & 0.00000 & -1.42003 & 0.00000 \\
$\mathrm{C}$ & -1.23502 & -0.68053 & 0.00000 \\
$\mathrm{C}$ & 1.23502 & -0.68053 & 0.00000 \\
$\mathrm{C}$ & 0.00000 & 2.81981 & 0.00000 \\
$\mathrm{C}$ & 0.00000 & -2.81983 & 0.00000 \\
$\mathrm{C}$ & -1.20898 & 3.56632 & 0.00000 \\
$\mathrm{C}$ & 1.20896 & 3.56631 & 0.00000 \\
$\mathrm{C}$ & -1.20901 & -3.56628 & 0.00000 \\
$\mathrm{C}$ & 1.20899 & -3.56628 & 0.00000 \\
$\mathrm{~N}$ & -2.20810 & 4.16937 & 0.00000 \\
$\mathrm{~N}$ & 2.20811 & 4.16938 & 0.00000 \\
$\mathrm{~N}$ & -2.20811 & -4.16938 & 0.00000 \\
$\mathrm{~N}$ & 2.20812 & -4.16938 & 0.00000 \\
$\mathrm{H}$ & -2.17673 & 1.23005 & 0.00000 \\
$\mathrm{H}$ & 2.17673 & 1.23005 & 0.00000 \\
$\mathrm{H}$ & -2.17673 & -1.23005 & 0.00000 \\
$\mathrm{H}$ & 2.17673 & -1.23005 & 0.00000 \\
\hline \hline
\end{tabular}

TABLE VII. PBE-optimized geometry of TCNE in cartesian coordinates and $\AA$.

\begin{tabular}{lrrr}
\hline \hline TCNE & & & \\
\hline $\mathrm{C}$ & 0.0000 & 0.6901 & 0.0000 \\
$\mathrm{C}$ & 0.0000 & -0.6901 & 0.0000 \\
$\mathrm{C}$ & 1.21464 & 1.4330 & 0.0000 \\
$\mathrm{C}$ & 1.21464 & -1.4330 & 0.0000 \\
$\mathrm{C}$ & -1.21464 & 1.4330 & 0.0000 \\
$\mathrm{C}$ & -1.21464 & -1.4330 & 0.0000 \\
$\mathrm{~N}$ & 2.21911 & 2.0231 & 0.0000 \\
$\mathrm{~N}$ & 2.21911 & -2.0231 & 0.0000 \\
$\mathrm{~N}$ & -2.21911 & 2.0231 & 0.0000 \\
$\mathrm{~N}$ & -2.21911 & -2.0231 & 0.0000 \\
\hline \hline
\end{tabular}

[1] H. Alves, A. S. Molinari, H. Xie, and A. F. Morpurgo, Nat. Mat. 7, 574 (2008).

[2] S. Gunes, H. Neugebauer, and N. S. Sariciftci, Chem. Rev. 107, 1324 (2007).

[3] G. Sini, J. S. Sears, and J.-L. Brédas, J. Chem. Theor. Comp. 7, 602 (2011).

[4] O. T. Hofmann, V. Atalla, N. Moll, P. Rinke, and M. Scheffler, New J. Phys. 15, 123028 (2013).

[5] J. F. Janak, Phys. Rev. B 18, 7165 (1978).

[6] L. Hedin, Phys. Rev. 139, A796 (1965).

[7] W. G. Aulbur, L. Jönsson, and J. W. Wilkins, Solid State Phys. 54, 1 (1999).

[8] G. Onida, L. Reining, and A. Rubio, Rev. Mod. Phys. 74, 601 (2002).

[9] P. Rinke, A. Qteish, J. Neugebauer, C. Freysoldt, and M. Scheffler, New J. Phys. 7, 126 (2005).

[10] M. S. Hybertsen and S. G. Louie, Phys. Rev. B 34, 5390 (1986).

[11] F. Caruso, P. Rinke, X. Ren, M. Scheffler, and A. Rubio, Phys. Rev. B 86, 081102(R) (2012).

[12] J. P. Perdew, K. Burke, and M. Ernzerhof, Phys. Rev. Lett. 77, 3865 (1996)

[13] J. P. Perdew, M. Ernzerhof, and K. Burke, J. Chem. Phys. 105, 9982 (1996).

[14] J. Paier, B. G. Janesko, T. M. Henderson, G. E. Scuseria, A. Grneis, and G. Kresse, J. Chem. Phys. 132, 094103 (2010).

[15] A. Grüneis, G. Kresse, Y. Hinuma, and F. Oba, Phys. Rev. Lett. 112, 096401 (2014).

[16] X. Ren, M. Noa, F. Caruso, M. Scheffler, and P. Rinke, (unpublished).

[17] F. Caruso, P. Rinke, X. Ren, A. Rubio, and M. Scheffler, Phys. Rev. B 88, 075105 (2013).
[18] V. Blum, R. Gehrke, F. Hanke, P. Havu, V. Havu, X. Ren, K. Reuter, and M. Scheffler, Comp. Phys. Comm. 180, 2175 (2009).

[19] X. Ren, P. Rinke, V. Blum, J. Wieferink, A. Tkatchenko, A. Sanfilippo, K. Reuter, and M. Scheffler, New J. Phys. 14, 053020 (2012).

[20] I. Y. Zhang, X. Ren, P. Rinke, V. Blum, and M. Scheffler, New J. Phys. 15, 123033 (2013).

[21] V. Atalla, P. Rinke, I. Zhang, O. T. Hofmann, and M. Scheffler, (unpublished).

[22] V. Atalla, M. Yoon, F. Caruso, P. Rinke, and M. Scheffler, Phys. Rev. B 88, 165122 (2013).

[23] T. Körzdörfer and N. Marom, Phys. Rev. B 86, 041110 (2012).

[24] D. L. Lichtenberger, R. L. Johnston, K. Hinkelmann, T. Suzuki, and F. Wudl, J. Am. Chem. Soc. 112, 3302 (1990).

[25] B. Milián, R. Pou-Amérigo, R. Viruela, and E. Ortí, Chem. Phys. Lett. 391, 148 (2004).

[26] N. Marom, F. Caruso, X. Ren, O. T. Hofmann, T. Körzdörfer, J. R. Chelikowsky, A. Rubio, M. Scheffler, and P. Rinke, Phys. Rev. B 86, 245127 (2012).

[27] T. Stein, H. Eisenberg, L. Kronik, and R. Baer, Phys. Rev. Lett. 105, 266802 (2010).

[28] K. N. Houk and L. L. Munchausen, J. Am. Chem. Soc. 98, 937 (1976).

[29] I. Ikemoto, K. Samizo, T. Fujikawa, K. Ishii, T. Ohta, and H. Kuroda, Chem. Lett. 3, 785 (1974).

[30] D. Dougherty and S. P. McGlynn, J. Am. Chem. Soc. 99, 3234 (1977).

[31] T. Kobayashi, Z.-I. Yoshida, H. Awaji, T. Kawase, and S. Yoneda, Bull. Chem. Soc. Jpn. 57, 2591 (1984).

[32] H. Stafast and H. Bock, Tetrahedron 32, 855 (1976).

[33] D. Dougherty, K. Wittel, J. Meeks, and S. P. McGlynn, J. Am. Chem. Soc. 98, 3815 (1976). 